\title{
Elasticity of Floppy and Stiff Random Networks
}

M. Wyart, H. Liang, A. Kabla, and L. Madadevan, Physical Review

Letters 101, 215501 (2008).

\section{Recommended with a commentary by Alex Levine, UCLA}

\section{Introduction}

Understanding the mechanics of periodic solids is one of the fine achievements of condensed matter physics. Periodicity is the key to developing a tractable solution to the problem of the dynamics of a (possibly infinite) lattice of masses coupled by harmonic springs. The reader can find an excellent discussion of this problem in Solid State Physics by Ashcroft and Mermin [1]. A broader discussion of the generalized elasticity of liquid crystals and elastic solids is found in Principles of condensed matter physics by Chaikin and Lubensky [2], and, here too, one relies on translational symmetries imposed by periodic structures. What happens when one breaks these rules to consider the elasticity of disordered condensed states of matter? In this letter, M. Wyart and collaborators explore such a problem, which presents fundamentally interesting theoretical issues, and has relevance to understanding the mechanics of a wide variety of systems, including covalent glasses, such as those composed of Silicon or Germanium [3, 4], granular matter, and even filament networks such as those found in the cytoskeleton of eukaryotic cells.

To begin a discussion of the mechanics of amorphous solids, it is helpful to recall Maxwell's criterion for rigidity in a network of point-like nodes coupled by central forces. The question to be addressed is how many such interactions between nodes are necessary to ensure that the system is rigid. By rigid, we mean that there are no deformation modes of the network, other than its rigid body translations and rotations, that incur zero energy cost. In the literature, such nontrivial zero energy modes of deformation are called "soft," while those modes that cost finite energy are "rigid." Given that there are $N$ nodes in $d$ dimensions, we see immediately that there are $N d$ degrees of freedom for the system. The criterion of a zero energy deformation sets $N_{c}$ constraints on these degrees of freedom since that number of interparticle distances must not change under a soft deformation. If there are, on average, $z$ interactions between a node and its neighbors, there are

$$
N_{c}=\frac{z N}{2}
$$


constraints. The factor of one-half comes from that fact that each interaction is shared by two nodes. Each constraint reduces by one the number of free degrees of freedom. Thus, the constraints leave $d N-N_{c}$ free degrees of freedom. Combining this result with Eq. 1 and neglecting the small [ $d(d+1) / 2$ ] number of rigid body motions of the entire system of $N$ particles, we see that when $z<z_{c}=2 d$, there will be remaining under-constrained and thus soft modes of the system. For $z>z_{c}$ we expect there to be no such floppy modes. A system at the boundary $z=z_{c}$ is referred to as isostatic.

The authors performed a numerical experiment on two dimensional networks of about $10^{4}$ particles connected by harmonic springs and brought near the isostatic limit. To make the network, they compressed (numerically) a set of (bidisperse, in order to avoid crystallization) disks. When these disks were jammed, they were replaced by nodes at their centers. These nodes are then connected to a subset of their nearest neighbors by harmonic springs so that $z \approx 5.5$. Then the network is pruned so that $z$ is reduced toward the isostatic point $z=4$.

\section{The scaling properties of the stress strain rela- tion}

The authors examine the shear stress (nondimensionalized using the spring constants of the internode springs) as a function of the applies shear strain $\gamma$. They do this on both sides of the isostatic point by examining the stress strain relation for both floppy networks with soft modes $\left(\delta z=z-z_{c}<0\right)$ and stiff ones $(\delta z>0)$ that do not. For the floppy networks, there is a strongly non-Hookean collective response of the network to applied strain: Below a certain critical value of the strain $\gamma^{\star}$ there is no stress, as one would expect for a system that can accommodate the imposed deformation via soft modes that do not store elastic energy. They find a scaling relation

$$
\gamma^{\star} \sim|\delta z|^{\beta} .
$$

Note the correspondence of this notation to the standard treatment of the scaling of the order parameter field with reduced temperature at a second order critical point. Here $\gamma^{\star}$ plays the role of the order parameter and the distance from the isostatic point parameterized by $\delta z$ plays the role of the reduced temperature. As suggested by Eq. $2 \gamma^{\star}$ goes to zero continuously as one approaches the isostatic point from below, $\delta z \rightarrow 0^{-}$. At the isostatic point, they find that the system responds nonlinearly to applied strain with the stress growing parabolically: $\sigma \sim \gamma^{2}$. 
Following up on the analogy to critical phenomena, the authors propose and provide convincing evidence that the stress-strain relations for systems near the isostatic point can be collapsed onto a single universal curve

$$
\sigma=|\delta z| \gamma f_{ \pm}\left(\frac{\gamma}{|\delta z|}\right),
$$

with $f_{ \pm}(x)$ scaling functions above and below the isostatic point. The key point is that the onset of a collective elastic response of the network as $\delta z$ is increased through zero occurs via a second order critical point (at zero temperature), with all of its attendant scaling properties.

\section{The displacement field in floppy and isostatic networks}

The authors move on to discuss the nature of the displacement field in the strained network. In a uniform elastic continuum, one expects to observe affine deformation in response to uniformly applied strain at the boundaries. If one where to take an elastic rectangle and, by moving the top boundary parallel to itself, deform it into a parallelogram, one would expect any internal rectangle of elastic material to deform into a similarly deformed parallelogram. Under these circumstances, the displacement field in elastic equilibrium is expected to be homogeneous in space. In Fig. 1b the authors show the displacement field of a uniformly strained isostatic network. It is anything but uniform! There are large nonaffine deformations.

The authors consider a new displacement field that subtracts off the expected (and uniform) affine displacements of the nodes: $\delta R_{\mathrm{n} \text {.a. }}^{i}=R^{i}-R_{\text {affine }}^{i}$ is the vector representing the nonaffine part of the displacement of particle $i$. They compute from this a (pseudo-)scalar quantity, $\delta V_{\text {n.a. }}$ representing the local rotation of nearest neighbor springs under the imposed shear strain. Remarkably, this quantity diverges at the isostatic point:

$$
\delta V_{\text {n.a. }} \sim \frac{1}{\sqrt{|\delta z|}} .
$$

At first this seems quite odd. As one approaches a stiff network $\left(\delta z \rightarrow 0^{-}\right)$, there are larger and larger local rotations associated with the nonaffine motion of the system. The argument made by the authors is that larger nonaffine deformations are indeed needed to accommodate the imposed strain as the floppy network becomes more constrained. The rigid parts of the network act like a lever to generate these motions. The authors also present an elegant argument to understand this observation for networks with $\delta z>0$. 


\section{Summary}

There are two key points made in this letter. The first, based on their numerical explorations, is that stiff and floppy networks (on either side of the isostatic point) are controlled by a single second order critical point that can be characterized by two independently determined critical exponents. Secondly, these two exponents which describe the nonaffine nature of the deformation field and the elastic modulus control all other elastic properties of the material near the isostatic point. These results have profound implications for organizing a general framework for thinking about the mechanics of disordered media near the isostatic point, and have implications for a number of physical systems of current interest.

\section{References}

[1] N.W. Ashcroft and N.D. Mermin, Solid State Physics (Thomson Learning Co., New York, 1976). See Chapters 21 and 22.

[2] P.M. Chaikin and T.C. Lubensky, Principles of condensed matter physics (Cambridge University Press, Cambridge, 1995). See Chapter 6.

[3] W.H. Zachariasen, J. Am. Chem. Soc. 54, 3841 (1932).

[4] M.F. Thorpe, J. Non-Cryst. Solids 5, 365 (1983). 